\title{
Collaborative understanding of disciplinary correlations using a low-fidelity physics based aerospace toolkit
}

\author{
Erwin Moerland ${ }^{1}$, Richard-Gregor Becker ${ }^{2}$ and Björn Nagel ${ }^{1}$ \\ German Aerospace Center (DLR) \\ ${ }^{1}$ Institute of Air Transportation Systems, Hamburg, Germany \\ ${ }^{2}$ Institute of Propulsion Technology, Cologne, Germany
}

Keywords: Collaborative Design, Multidisciplinary Design and Analysis, Knowledge

Management, CPACS, RCE

Nomenclature

$\begin{array}{ll}\text { CPACS } & \begin{array}{l}\text { Common Parametric Aircraft } \\ \text { Configuration Scheme }\end{array} \\ \text { RCE } & \text { Remote Component Environment }\end{array}$

\begin{abstract}
Covering all relevant physical effects and mutual influences during aircraft preliminary design at a sufficient level of fidelity necessitates simultaneous consideration of a large number of disciplines. This requires an approach in which teams of engineers apply their analysis tools and knowledge to collaboratively approach design challenges.
\end{abstract}

In the current work, recent technical advancements of the German Aerospace Center (DLR) in data and workflow management are utilized for establishing a toolbox containing elementary disciplinary analysis modules. This toolbox is focussed on providing fast overall aircraft design capabilities. The incorporated empirical and physics based tools of low fidelity level can be used for setting up modular design workflows, tailored for the design cases under consideration. This allows the involved engineers to identify initial design trends at a low computational effort. Furthermore, areas of common physical affinity are identified, serving as a basis for communication and for incorporating tools of higher fidelity in later phases of the design process. Clear visualisation methods aid in efficiently translating knowledge between the involved engineers within the identified areas of common affinity.

A system-of-systems approach is established by applying the elementary aircraft design toolbox for the establishment of requirement catalogues for engine preliminary design. The engine designers at their turn deliver initial performance correlations for application in the aircraft design toolbox. In this way, a clear synergy is established between the design of both the airframe and power plant. Using this approach, engineers of different technical backgrounds share their knowledge in a collaborative design approach.

The use case guiding the present work involves a conventional short to medium range aircraft sent at half the design range. The wing area and aspect ratio are varied to investigate the influence on the engine requirements catalogue for this particular mission. 


\section{Introduction}

Aircraft design is a complex procedure, which involves an increasing amount of disciplines considered simultaneously. During recent years, Multidisciplinary Design \& Optimization (MDO) techniques have become state-of-the-art and are evolving continuously. Applications to design of novel aircraft are however only occasionally seen and wide exploitation of modular MDO processes at industry level is not yet clearly observed [1], [2]. Due to the large complexity of analysing the multitude of relations between involved design disciplines, the analysis of novel configurations cannot be handled by a single person anymore. Collaborative approaches in teams of specialists and integrators are required to master the challenge of understanding the relevant physical effects involved in the design of aircraft [3].

A lot of effort has been put in generating technical solutions to aid design teams in connecting their disciplinary analysis capabilities. The virtual extended enterprise as developed during projects VIVACE [4] and CRESCENDO [5] forms a tangible example of this development. Within these and similar projects, focus has been placed on exchanging explicit ${ }^{1}$ knowledge by using common data exchange formats and setting up technical design frameworks for interconnecting analysis codes. Aside this development, methods for collaboration in teams of engineers have also been investigated.

The Common Parametric Aircraft Configuration Scheme (CPACS) is an xml-based data model developed at the German Aerospace Centre (DLR), representing an explicit description of the aircraft in a structured manner. Aside a geometrical description of the vehicle, other

\footnotetext{
1 'Explicit' (or formal) knowledge: knowledge that can be captured in design rules, implicit (or tacit) knowledge: knowledge possessed by an individual, mostly based on experience, which is difficult to communicate via words and symbols [17].
}

relevant conceptual design data such as missions, fleets and airports are exchanged using CPACS [6]. With the parallel development of the Remote Component Environment (RCE) at DLR, a framework for connecting analysis tools on distributed servers has been created [7], using CPACS as interface.

The technical achievement of using frameworks for interconnecting analysis tools applying the aforementioned data exchange methods is showing large benefits. Experience gained during the DLR collaborative design projects "TIVA" and "VAMP" [8] however shows that operating a numerical analysis system in a team of specialists presents a large challenge of its own. Therefore, as also introduced by Kroo [9], a larger part of the research should focus on addressing challenges at the organizational level of MDO. The collaborative way of working, indicated as third generation in Fig. 1, is required to share implicit ${ }^{1}$ knowledge within the design team. The indicated shift in focus toward organizing effective collaboration among all involved engineers is however still in its early stages.

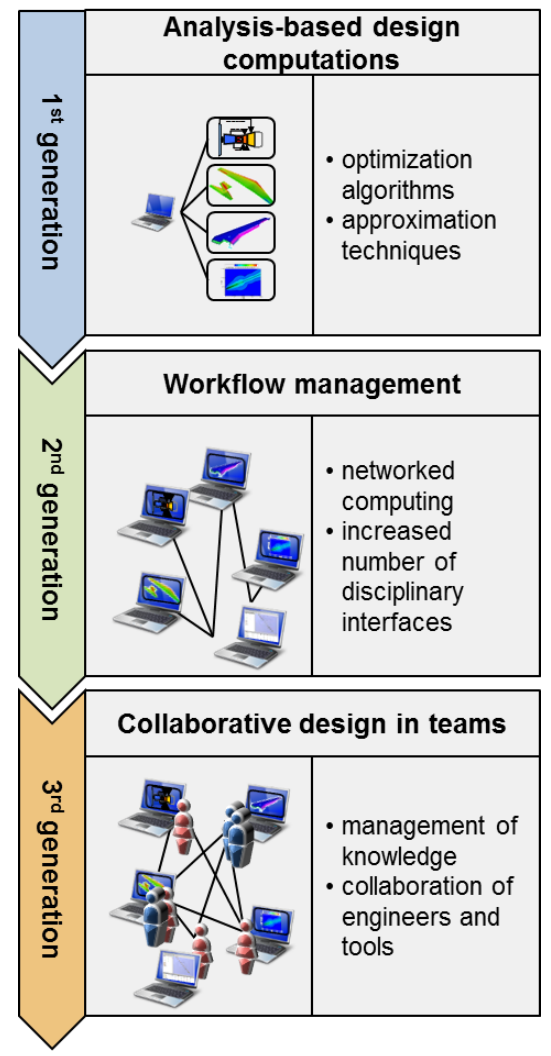

Fig. 1 Evolving generations in MDO 
The main question guiding the collaborative design effort is:

How to enable communication among engineers having different specialisms?

In the present study, it is investigated how multidisciplinary interactions and affinity for common disciplines can be identified and used as a basis for communication (see Fig. 2). Using a practical design problem, experience is gained on the needs to ensure effective collaborative approach in aerospace design teams. As indicatively shown in Fig. 2, common disciplinary affinity between knowledge bearers serves as starting point for comprehensible communication within the team. This area of common affinity can be defined by shared explicit knowledge, e.g.: design parameters exchanged between disciplines, but also by the less straightforwardly identifiable implicit knowledge, e.g.: common theoretical methods applied within the analysis codes.

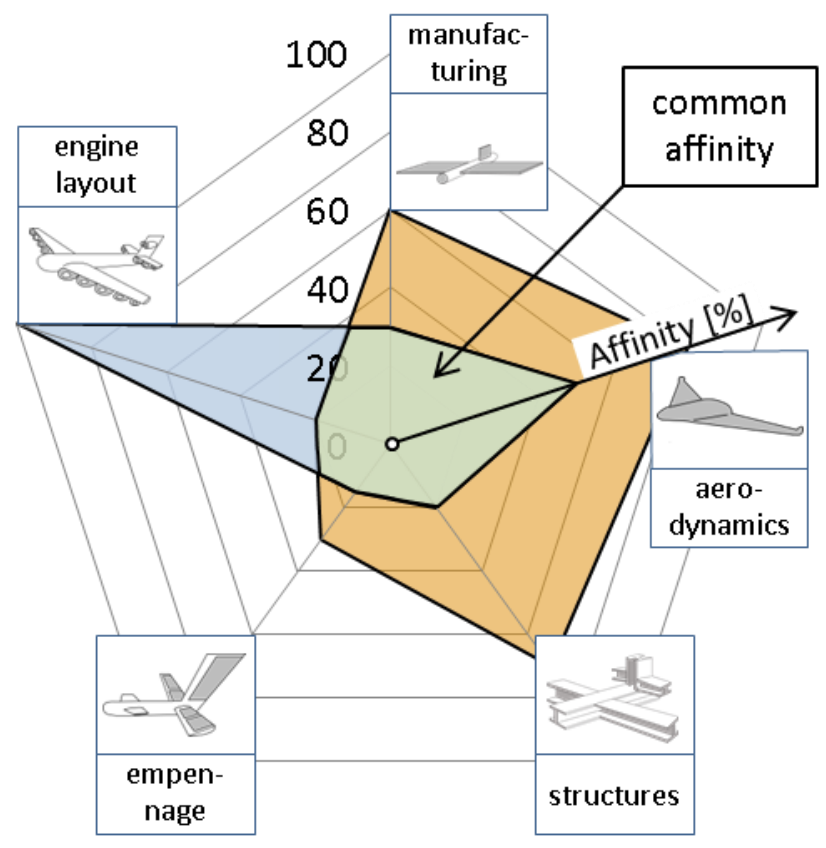

Fig. 2 Common affinity of two knowledge bearers serving as basis for communication.

- blue: pre-engine designer,

- orange: aircraft pre-designer,

- green: area of common affinity

\section{Low-fidelity toolkit for mutual understanding and knowledge transfer}

In a previous study, the authors investigated the possibility to perform a system-of-systems approach in aircraft design [3]. Defining the area of common affinity (see Fig. 2) between the involved engineers proved not to be straightforward. On the implicit level, this was mainly caused by the difference in engineering backgrounds of the involved parties. On the explicit level this was due to the difference in applied design methods and - practically - due to differences in applied data exchange formats.

For serving the assessment of the overall system under analysis, large commonality among the involved analysis tools is required. Furthermore, the incorporated tools (and maybe even engineers) should be modular in a sense that a change of analysis methods within the process requires only little effort. For the current investigation, a basic pool of low-fidelity physical analysis tools is created using the technical capabilities provided by the CPACS data exchange format and the integration framework RCE as a basis. The main goal of the modular system of analysis tools is to create the possibility to quickly identify physical effects and cross-disciplinary influences.

The studies at low fidelity level are used for identifying common knowledge affinity between the involved disciplines. After identifying these correlations, higher-level analysis modules can be incorporated in the design system to increase the certainty of the identified correlations. Since expert knowledge is required to interpret results of the overall system, this process tends to go beyond plainly connecting analysis tools and observing the results. Instead, a system of distributed low and high-level competencies is created.

The fidelity level of analysis modules used in aircraft design can be subdivided in four levels:

Level-0 tools are based on statistical or empirical design rules and allow exploration of the conventional design space only. 
Level-1 tools are based on a simplification of the physics of the design problem. These tools are applicable to simple extensions of the conventional design space and mostly involve physical behaviour of a linear nature.

Level-2 tools are based on accurate physical representations of the disciplines involved in the design problem: the geometrical representation is much more detailed; the physics underlying the analysis code is of high detail or a combination of the both. Tools of this level may be used for analysing unconventional designs.

Level-3 tools represent the most accurate simulation capabilities. These are used to capture detailed local effects and mostly do not allow for automation.

Tab. 1 Main properties of analysis modules, per fidelity level

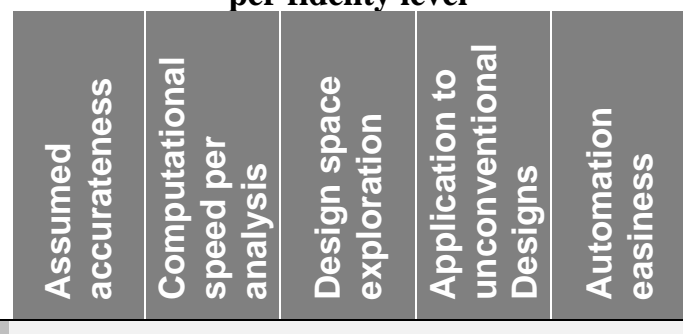

\begin{tabular}{|lcccccc|}
\hline Level-0 & - & ++ & + & no & ++ \\
Level-1 & - & + & ++ & $+/-$ & + \\
\hline Level-2 & + & - & - & ++ & - \\
\hline Level-3 & ++ & -- & -- & + & -- \\
\hline
\end{tabular}

The main properties of the analysis modules of different fidelity level are summarised in Tab. 1. The current work focuses on the interconnection of tools of level-1 fidelity in order to efficiently scan the design space at low calculation effort. The level-0 tool VAMPzero is used for initiating the aircraft as well as for closing the iterative design loop [10]. It calculates the aircraft properties for which level-1 analysis modules are currently still under development.

After analysing physical properties of the aircraft concept model, its 'goodness' is evaluated according to the requirements set in the initiation phase of the concept assessment. As can be seen in Fig. 3, within such an evaluation again multiple disciplines are represented. In the current study the aircraft costs are analysed using a low-level DOC calculation module. Climate impact, as well as noise and capacity assessment is part of future work.

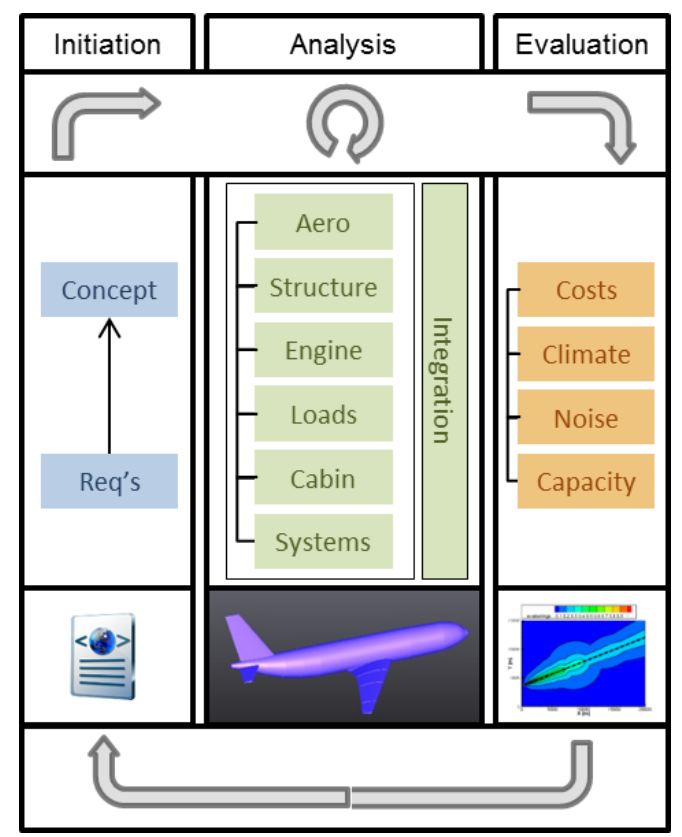

Fig. 3 Phases in aircraft concept assessment

All tools within the toolkit under development, both in the analysis as in the evaluation category, include a connection to the central data exchange format CPACS. The tools are therefore modularly applicable; the user can for example choose to exchange individual analysis modules using different approaches or of different fidelity level. The modules making up the analysis toolkit are hosted on multiple dedicated servers and analyses can be triggered using the RCE framework.

To provide the workflow integrator ${ }^{2}$ clear and concise information on the analysis modules

2 As elaborated in [3], design teams within multidisciplinary design approaches ideally consist of one or more workflow integrators connecting all involved analysis modules to logical design workflows, supported by the specialists individually interpreting the results of their disciplinary modules. An operator takes care of the overall course of action within the design process. 
within the toolkit, a standard has been developed for connecting the tools to the RCE framework. This tool wrapping plainly consists of a standard folder structure to be used for input and output data, as well as scripts for encapsulating the tool. These scripts trigger the actual calculation and control tool execution behaviour. Furthermore, the end user is provided a well-balanced amount of status information through filtering the often excessive output information for main output messages. The excessive calculation logbooks can be used by the specialist for debugging purposes on the dedicated server when a tool does not provide the intended results.

The main purpose of having a standard for tool wrapping is generating the possibility for flexible application to a multitude of design questions and aircraft configurations. From experience it is found that this collaborative approach requires a change in mind-set of the developing engineer: (s)he needs to be constantly aware of how external users without the experience of a disciplinary specialist will try to approach the tool at hand and clearly define its application boundaries. In this design for collaboration approach, putting large effort in writing a proper wrapping code generally saves lot of time during the application period of the module. This wrapping code has to provide the end user with clear information on assumptions, warnings and errors encountered during tool execution. Assumptions and warnings need to be built up to flexibly react on the contents of the provided input.

\section{Simultaneous aircraft and engine design}

As can be seen from Fig. 2, the area of common affinity between aircraft and engine designers is relatively small. This is also seen in the industry, in which the airframe and engines are often designed by separate parties. At the DLR, two parallel projects for aircraft and engine preliminary design are currently executed. The design cycles are synchronised in such a way, that the aircraft design workflow provides design points for the design of the corresponding engine. The generated engine performance data is at its turn used to determine the performance of the complete integrated airframe.

In light of these projects, the current study encompasses the generation of an aircraft analysis workflow aimed at generating request for proposal (RFP) documents for the layout of a corresponding engine concept. Fig. 4 shows an N2-chart of the connections between the lowlevel tools as used within the analysis, as well as the purpose and name of each tool. This chart has been established through communication with all specialists that programmed the individual analysis modules. Since within the actual tool connections data of an explicit nature is exchanged, this step in the setup of the analysis workflow will be aided by automatic identification of required input data in future work. This will allow for more time to be spent on exchanging implicit knowledge, e.g.: on the appropriateness of a tool to generate required input data.

After identifying the required input and available output of each analysis module, the N2-chart aids in logically ordering the workflow in an initiation, iterative and evaluation phase.

The application of CPACS as central data exchange format considerably reduces the required amount of actual connections between the modules within the RCE framework, since information of consecutively executed modules is appended to this single data file.

To reduce complexity in the analysis workflow, the engine is represented by a database containing pre-calculated performance data. The database tables are created by performing thermodynamic analyses of the engine cycle at a multitude of operating points. Therein, the underlying engine deck is fixed in terms of principle cycle parameters such as turbine entry temperature (TET), overall pressure ratio (OPR) and fan pressure ratio (FPR). However, using 'rubber engine' scaling principles, the available engines can be scaled in mass flow by $+/-20 \%$. 


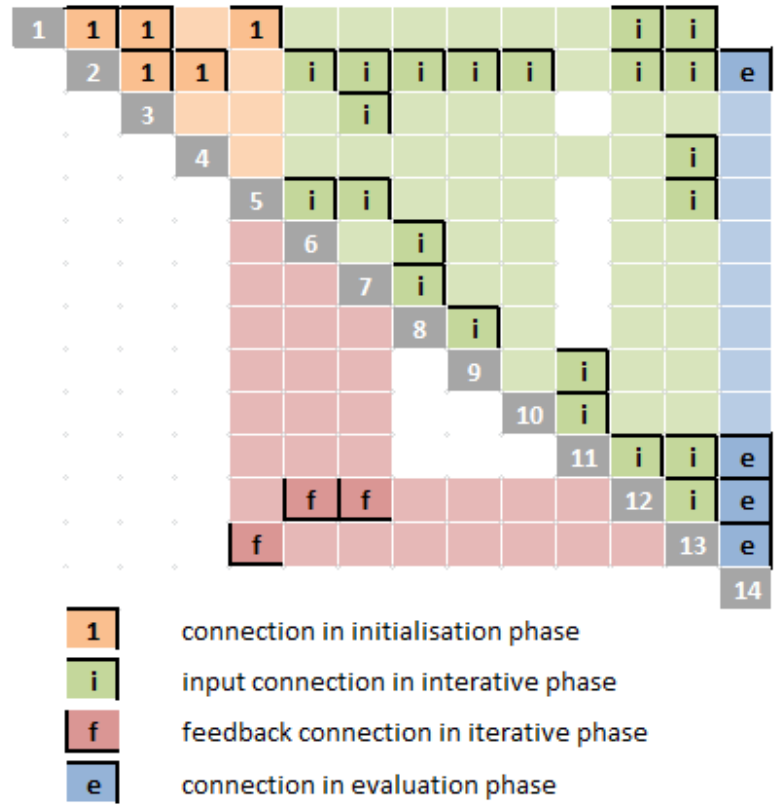

\begin{tabular}{lll}
$\#$ & purpose & tool \\
\hline 1 & TLAR \& basic aircraft geometry & user input \\
\hline 2 & geometry variation & GPP \\
\hline 3 & mass initialisation & VAMPzero \\
\hline 4 & aerodynamic performance map & Tornado \\
\hline 5 & engine mass \& performance map & TWDat \\
\hline 6 & loadCase determination & LCG \\
\hline 7 & weight and balance & WandB \\
\hline 8 & spanwise loading & TRIM_VL \\
\hline 9 & wing primary mass & PESTwing \\
\hline 10 & wing secondary mass & PESTsewi \\
\hline 11 & mass tree update & CMU \\
\hline 12 & aircraft mass synthesis & VAMPzero \\
\hline 13 & fuel mass and engine scaling & FSMS \\
\hline 14 & direct operating costs & DOC
\end{tabular}

Fig. 4 N2-Chart providing the connections between analysis modules in the aircraft analysis workflow

The more the engine differs from its validated unscaled basic thermodynamic cycle, the more care has to be taken in interpreting the corresponding performance data. In Fig. 5, an engine performance map as read out from TWDat and interpreted by the mission simulation module FSMS is shown. As concluded during the design studies, the current simplified representation however has its limitations: no engine ratings are included, allowing the aircraft engineer to theoretically let the aircraft fly at full thrust throughout the entire design mission. In setting up a workflow involving engine data, the aircraft engineer needs to provide the engine designer the intended mission data of the airframe in order to attain proper performance data coverage.

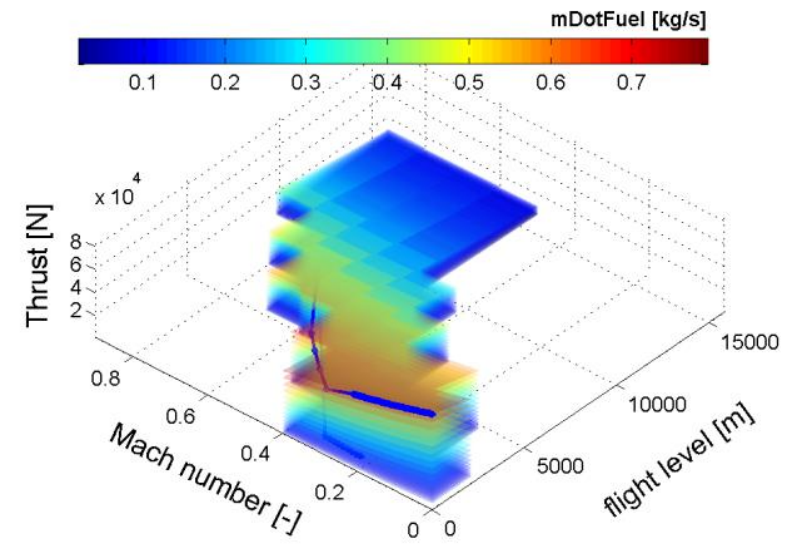

Fig. 5 Interpolation within the performance map of the CFM56 engine. Blue line: interpolation trajectory throughout the simulated mission

A resulting engine requirements catalogue for the short- to medium range A320-like reference aircraft 'D150' is shown in Tab. 2. The mission simulation tool FSMS is adjusted to specifically calculate the following design points for the catalogue:

One Engine Inoperative (OEI) condition determines the required engine scaling factor according to Certification Specifications chapter 25.121 [11]. For a fly out manoeuvre with engine failure exactly occurring at decision speed V1, the engine is scaled such that the minimum fly out gradient and velocity are attained by the aircraft.

End of Field (EOF) is the condition with the largest fly out climb angle, reached shortly after the ground run. This is generally the point with the highest shaft speed and turbine inlet temperature requirements.

Mid Cruise (MCR) is used as the aerodynamic design point providing the highest component efficiencies for minimizing engine specific fuel consumption.

Top of Climb (TOC) is the point just before the aircraft starts its cruise phase, used to 
determine the maximum non-dimensional engine performance parameters, such as corrected component mass flows and speeds.

Once the engine performance data is calculated, an additional database entry in TWDat can be added to verify its correspondence to the established requirements. This at its turn might lead to an update of the requirements catalogue.

Tab. 2 Engine design point data for the requirements catalogue [a/c: D150, engine: CFM56-5A5, des. range: $1800 \mathrm{~nm}$ ]

\begin{tabular}{|c|c|c|c|c|c|}
\hline & & 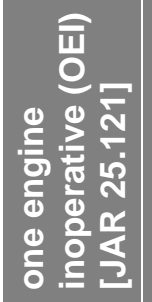 & 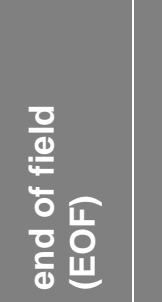 & 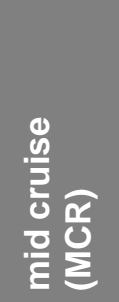 & 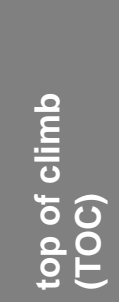 \\
\hline $\mathrm{T}_{\text {required }}$ & {$[\mathrm{N}]$} & 82383 & 78327 & 19936 & 26973 \\
\hline $\mathrm{T}_{\text {delivered }}$ & {$[\mathrm{N}]$} & 80880 & 78327 & 19936 & 26973 \\
\hline time & [m':s"] & 0'50" & 0'45"' & $139^{\prime}$ & $29.6^{\prime}$ \\
\hline altitude & {$[\mathrm{m}]$} & 64 & 45 & 10376 & 10000 \\
\hline Mach & {$[-]$} & 0.23 & 0.26 & 0.78 & 0.78 \\
\hline$\alpha$ & [deg] & 9.4 & 8.0 & 4.1 & 3.6 \\
\hline Y & [deg] & 2.4 & 8.0 & 0.0 & 1.1 \\
\hline$\theta$ & [deg] & 11.8 & 16.0 & 4.1 & 4.7 \\
\hline $\mathrm{dT}_{\text {ISA }}$ & [deg] & 0 & 0 & 0 & 0 \\
\hline rating & {$[-]$} & OEI & MTO & MCR & MCL \\
\hline ECS & {$[-]$} & on & on & on & on \\
\hline WAI & {$[-]$} & on & off & off & off \\
\hline HPX & {$[-]$} & tbd & tbd & tbd & tbd \\
\hline $\mathrm{nPax}$ & {$[-]$} & 150 & $\mathrm{H}_{\mathrm{OEImax}}$ & {$[\mathrm{m}]$} & tbd \\
\hline nEng & {$[-]$} & 2 & $\mathrm{H}_{\text {airport }}$ & {$[\mathrm{m}]$} & 0 \\
\hline$t_{\text {climb }}$ & [m'] & $28^{\prime}$ & MTOM & {$[\mathrm{t}]$} & 73500 \\
\hline TOFL & {$[\mathrm{m}]$} & 2120 & MLM & {$[\mathrm{t}]$} & 64500 \\
\hline Vappr & {$[\mathrm{m} / \mathrm{s}]$} & tbd & ESF & {$[-]$} & 1.019 \\
\hline
\end{tabular}

ECS: env. control system, WAI: wing anti-icing, HPX: eng. power off take, ESF: eng. scaling factor

Using the N2 Chart (Fig. 4), the required modules are connected using the CPACS data format within the integration framework RCE. Fig. 9 shows the resulting workflow, specifically aimed at generating requirement catalogues for engine predesign. After initializing the aircraft geometry according to the design of experiments study at hand (see section 4), an iterative loop is started in which the engine scaling factor is brought to convergence. In the current setup, the wing and engine mass is determined using level-1 tools, whereas the other aircraft masses are determined using VAMPzero. Modules having no direct input connection as identified in the N2-chart (Fig. 4) are executed in parallel to save calculation time. After reaching convergence, the engine requirements catalogue is obtained for the configuration under investigation.

As already stated in section 2, in setting up such an analysis workflow, the need for a balanced combination of workflow integrators with general knowledge in connecting the specialists' tools on the one hand and disciplinary specialists on the other hand is clearly observed. The specialists need to ensure the connected tool is used properly and results are interpreted in a proper way, whereas the workflow integrator should provide general knowledge for the integration in analysis workflows and question the generated overall results at hand.

\section{Design Study: influence of wing planform on engine scaling requirement}

In the present work, it is chosen to keep the fidelity level of the applied tools low enough to provide relatively quick calculation results, although modelling the effects to be studied with physical relations. The workflow for the design study, depicted in Fig. 9 can be divided in six main parts.

The initiator part will use a geometric preprocessor to adjust the baseline aircraft geometry in CPACS. For the current study, a predefined geometrical description of the D150 aircraft is applied. As indicated in Fig. 6, the wing area and aspect ratio are varied around the baseline values of the D150 aircraft. VAMPzero is used to obtain a first mass estimation and in parallel, the aerodynamic performance map is generated using Mach, Reynolds and angle of attack sweeps in the vortex-lattice programme Tornado [12]. 
After the initiation, an engine performance map and weight is loaded from TWDat (see section 3 ) and in two parallel branches, the wing primary and secondary masses are estimated. Within the tools PESTwing and PESTsewi, a beam model representation for main wing structure sizing and empirical relations for secondary structure mass estimation are applied. The required wing loading is determined using a trimming routine incorporating a connection the vortex-lattice code AVL [13]. To complete the aircraft mass determination, VAMPzero is again used to estimate the aircraft masses not belonging to the wing group.

Knowing the aircrafts aerodynamic, engine and mass properties, the design mission is flown using the mission simulator FSMS to obtain the fuel requirements and the required engine scaling factor. Aside required fuel mass, payload-range diagrams as well as emission values are calculated, and the requirements catalogue for the new engine is provided (see section 3$)$.

The determination of aircraft masses and engine scaling factor is iteratively performed, until the scaling factor converges and the engine required for the investigated configuration is obtained.

Within the concept evaluation, the direct operating costs are determined, after which the DOE is continued.

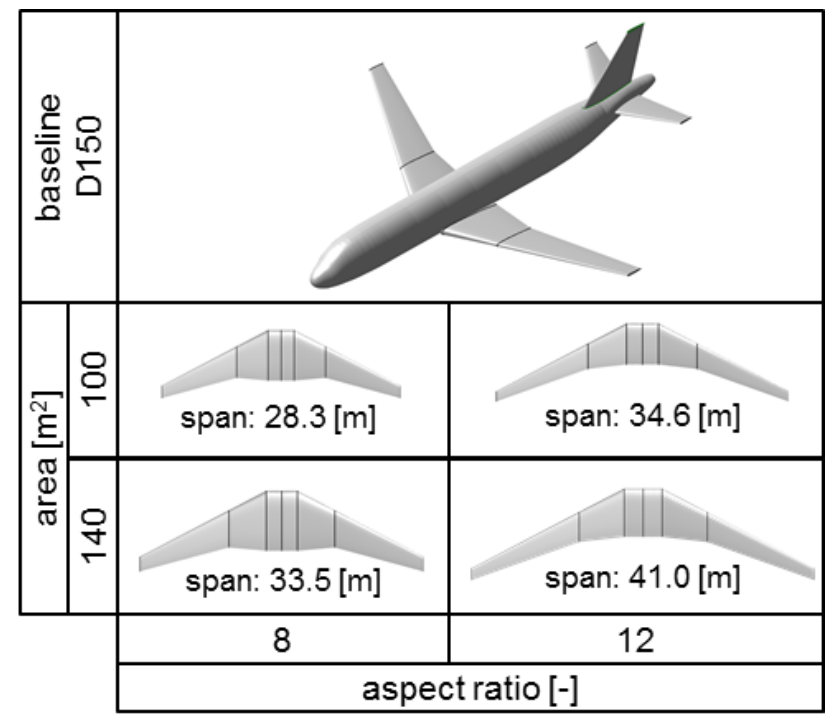

Fig. 6 Geometry changes within the performed design of experiments. Wing area: $100-140\left[\mathrm{~m}^{2}\right]$, aspect ratio: 8-12 [-].
Applying proper visualization methods aids considerably in establishing clear interdisciplinary communication among involved engineers. In [14], the usage of "level transfer functions" to assist in communicating physical relationships among the parties involved in a design exercise is suggested. Transfer functions are used for communicating metrics on one design level to understandable research objectives for another level. Such plots provide a "feel" for the involved engineers on how known geometrical parameters influence higher-level objectives.

Fig. 7 shows the required engine scaling factor and aircraft operating empty mass (OEM) for the studied geometric parameters. For the D150 reference aircraft, a scaling factor close to 1.0 is obtained. The difference is caused by the lowlevel physics being used in the workflow. A technology factor of 1.069 is used to correct the determined wing mass within the workflow to its known baseline value for the reference aircraft. It can be concluded that the aircraft with a slender wing and low wing area has the least stringent requirement on engine performance. The classical opposing aeroelastic correlation is seen when combining parts (a) and (b) of the figure: a slender wing leads to better aerodynamic efficiency (and thereby a low engine scaling factor), however the aircraft mass increases due to the large structural loads imposed by such a configuration.

Fig. 8 shows a resulting performance correlation for the aircraft. Within this level transfer function, the influence of the performance measures wing loading (W/S) and thrust-toweight ratio (T/W) on fuel requirements is shown for the geometries with correspondingly scaled engine. The boundaries of the T/W-W/S area are a consequence of the parameter variation chosen within the current study. 
(a): thrust scaling factor (below)

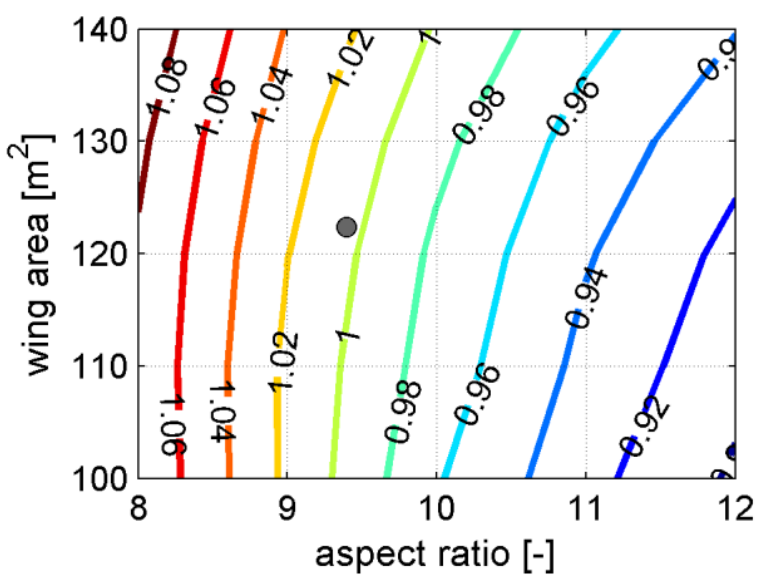

(b): operating empty mass (below)

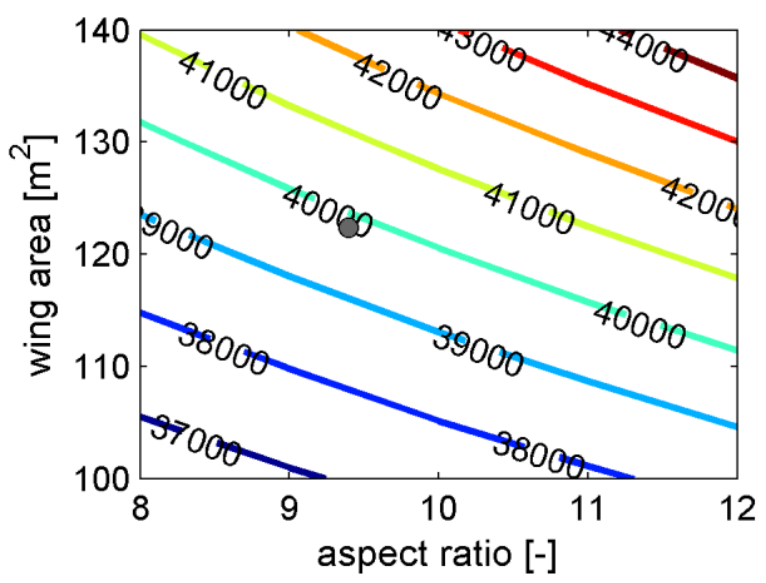

Fig. 7 Disciplinary parameter transfer plot: the influence of wing area and aspect ratio on engine scaling requirements (a) and aircraft empty mass (b)

[D150 reference aircraft indicated by grey dot]

litres of fuel per 100 seat kilometres (I/100skm)

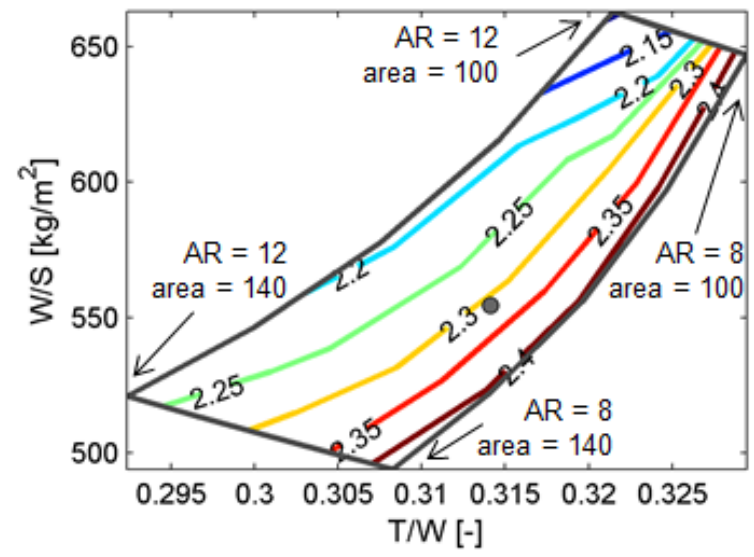

Fig. 8 Level transfer function showing relative effect on litres of fuel per 100 seat-kilometres for changing wing loading and thrust-to-weight ratio

[D150 reference aircraft indicated by grey dot]
The data represented within the figures above are obtained using a scalable database entry in the engine performance database TWDat. When a team of engineers chooses to further investigate a specific design point, a new data deck should be generated for the scaled engine at hand, in order to improve the accuracy of the results. For this the requirements catalogue as in Tab. 2 can be used.

In future work, correlations like the ones shown in Fig. 7 and Fig. 8 will be used as a basis for communication within teams of engineers. Disciplinary dependencies can be identified and the decision making process is supported by clarifying visualisations. After extending the toolkit with physical analysis modules for disciplines not yet covered, the design space is extended by considering less conventional aircraft configurations. Instead of using a predefined geometrical description of the reference aircraft in CPACS, an aircraft initiator based on knowledge-based engineering principles can be applied to attain a starting configuration [15].

When needed, connections to modules of higher fidelity level can be established using RCE, to cover the parts of the underlying physics that cannot be handled by level-1 tools. 


\section{Conclusion}

Although the technical means to connect aircraft analysis modules are available, large potential for improvement is still found in the application of these modules within multidisciplinary analysis workflows. Methods aimed at efficiently translating knowledge between researchers of various backgrounds involved within aircraft predesign are currently under development. The current work investigated how multidisciplinary interactions and areas of common affinity might serve as initial basis for communication among engineers.

The generation of modularly applicable analysis components requires a change in attitude of the design engineer. It proves to be a large effort to program these components such that a wide variety of aircraft configurations can be analysed without the need for problem-specific tool adjustments. Furthermore, providing disciplinary specific output using visualisations and messages understandable for a widely oriented public, such as workflow integrators, requires major thoughts. Identifying areas of common affinity between the engineers involved forms a starting point for the latter issue.

An initial application of a low level toolkit for combining aircraft and engine predesign has been shown. In the future, the toolkit will be extended with more low-level physics based analysis tools and applied to generate visualisations of cross-disciplinary correlations. When operators, workflow integrators and specialists gather in design teams, these kind of visualisations aid in understanding each other's considerations and interests. The flexibility of arbitrarily connecting analysis modules facilitated by RCE allows the design team to investigate physical trends at a level of detail appropriate to the question at hand.

Once mutual understanding of physical correlations is created, initial design space extensions can be studied using the combined explicit and implicit knowledge of the involved design team members. Extending the design space requires careful analysis of tool results and applicability considerations, since results cannot directly be validated by comparison to familiar aircraft designs. Especially at this stage, clear and streamlined communication among engineers is of utmost importance.

\section{Outlook}

In future work, more level-1 modules will be incorporated within the toolkit. When developing these new modules, the modularity of its application in workflows specifically aimed at providing a quick answer to the design question at hand should always be kept in mind. Furthermore, the level-1 toolkit will serve as basis for incorporation of uncertainty considerations within the analysis modules. By adding uncertainty values to the results, the possibility to not only determine the 'goodness' of an aircraft concept or requirements catalogue, but also with which certainty such a statement can be made is established.

A continuation of simultaneous aircraft and engine design is foreseen. The workflow and toolkit will be used to investigate combined unconventional aircraft and engine concepts, among which a strut-braced wing configuration with counter-rotating open rotor (CROR) engine is anticipated. A semi-automated aircraft and engine concept analysis workflow is to be established by incorporating the thermodynamic performance analysis and preliminary engine design environment GTlab [16]. In contrast to the pre-calculated and scaled performance decks used in the present study, airframe and engine conceptual design processes will be directly coupled in order to find the optimum engine cycle parameters for a given set of airframe and mission requirements. This will bring collaboration among aircraft and engine specialists and integrators in predesign phases to a higher level. 


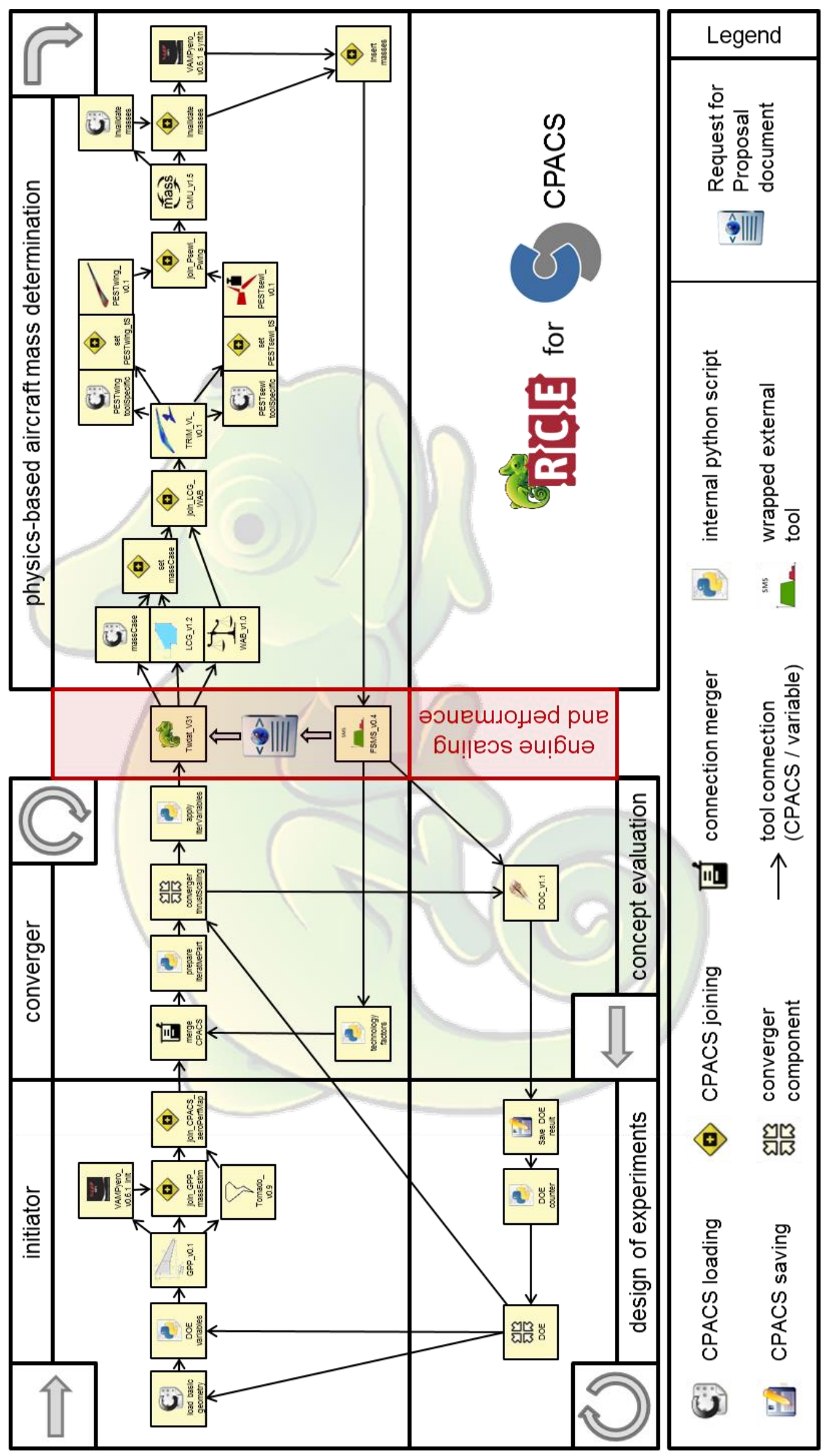

Fig. 9 Workflow of level-1 fidelity in RCE for CPACS 


\section{Acknowledgement}

The authors wish to thank Daniel Böhnke, Till Pfeiffer and Felix Dorbath for providing their tool contributions, bugs and help in resolving these in the aircraft analysis workflow.

\section{Contact details}

Erwin Moerland

German Aerospace Center (DLR)

Blohmstraße 18, 21079 Hamburg, Germany

Email: erwin.moerland@dlr.de

Telephone: +49-40-42878-4141

\section{References}

[1] G. La Rocca, "Knowledge based engineering techniques to support aircraft design and optimization," Doctoral Thesis, Delft University of Technology, Delft, The Netherlands, 2011.

[2] J. Agte, O. Weck, J. Sobieszczanski-Sobieski, P. Arendsen, A. Morris, and M. Spieck, "MDO: assessment and direction for advancement - an opinion of one international group," Structural and Multidisciplinary Optimization, vol. 40, no. 1-6, pp. 17-33, Apr. 2009.

[3] E. Moerland, T. Zill, B. Nagel, H. Spangenberg, H. Schumann, and P. Zamov, "Application of a distributed MDAO framework to the design of a short- to medium-range aircraft," 61th German Aerospace Congress (DLRK), Berlin, Germany, 2012.

[4] VIVACE Consortium, "VIVACE - Final Technical Leaflet,” 2007.

[5] CRESCENDO Consortium, "CRESCENDO Forum participants handbook,” 2012.

[6] C. Liersch and M. Hepperle, "A distributed toolbox for multidisciplinary preliminary aircraft design," CEAS Aeronautical Journal, vol. 2, no. 1, pp. 57-68, 2011.

[7] D. Seider, M. Litz, M. Kunde, R. Mischke, and P. Kroll, "RCE: Distributed, Collaborative Problem Solving Environment." 2012.

[8] B. Nagel, T. Zill, E. Moerland, and D. Böhnke, "Virtual Aircraft Multidisciplinary Analysis and Design Processes - Lessons Learned from the
Collaborative Design Project VAMP," The International Conference of the European Aerospace Societies (CEAS), Linköping, Sweden, 2013.

[9] I. Kroo, "Multidisciplinary Design Architectures : History and Status Optimization and Design," no. June. Stanford University, Palo Alto, CA, USA, 2006.

[10] D. Böhnke, B. Nagel, and V. Gollnick, "An Approach to Multi-Fidelity in Conceptual Airplane Design in Distributed Design Environments," IEEE Aerospace Conference, Big Sky, USA, 2011.

[11] European Aviation Safety Agency, "Certification Specifications and acceptable means of compliance for large aeroplanes CS-25, Amendment 13," 2013.

[12] T. Melin, "Tornado." [Online]. Available: http://www.redhammer.se/tornado/. [Accessed: 03-Jun-2013].

[13] M. Drela and H. Youngren, “AVL.” [Online]. Available: http://web.mit.edu/drela/Public/web/avl/. [Accessed: 24-Jul-2012].

[14] A. Hahn, "Providing Quantifiable Governance." NASA Technology Frontiers-ASH, 2010.

[15] P.-D. Ciampa, B. Nagel, and G. La Rocca, "Preliminary Design for Flexible Aircraft in a Collaborative Environment," The International Conference of the European Aerospace Societies (CEAS), Linköping, Sweden, 2013.

[16] R.-G. Becker, F. Wolters, M. Nauroz, and T. Otten, "Development of a gas turbine performance code and its application to preliminary engine design," 60th German Aerospace Congress (DLRK), Bremen, Germany, 2011.

[17] L. Prusak, "The Knowledge Notebook: What do we mean wen we say 'knowledge'?," Washington, pp. 44-45, 2006. 